\title{
Analytic Properties of Dispersion Curves for Efficient Eigenmode Analysis of Isotropic Waveguides using a Boundary Element Method
}

\author{
Dieter Dobbelaere, Hendrik Rogier, Daniël De Zutter \\ Electromagnetics Group, Department of Information Technology, Ghent University \\ Sint-Pietersnieuwstraat 41, Ghent, Belgium
}

\begin{abstract}
Recent developments in high-speed interconnects show a clear tendency towards higher bitrates, emphasizing the need for a reliable prediction of the waveguide behavior We present a frequency domain eigenmode analysis of isotropic waveguides using a boundary element method. Some properties of the dispersion curves can be beneficially leveraged into the numerical framework for calculating the waveguide characteristics as a function of frequency. The method allows the incorporation of highly conductive materials, which is demonstrated in a numerical example.
\end{abstract}

\section{INTRODUCTION}

Accurate prediction of waveguide characteristics, comprising modal dispersion curves and eigenmode profiles as a function of frequency, are primordial in the design of highspeed interconnects and waveguides. They predict all occuring wave phenomena, including crosstalk and signal losses. There is a large number of numerical techniques available for the eigenmode analysis of uniform waveguides [1], but we will only briefly discuss the two predominant methods.

On the one hand, finite difference and finite element techniques have been used to analyze dielectric waveguide structures [2], [3]. They allow an easy incorporation of inhomogeneous material regions, and the waveguide's propagation constants and eigenmode profiles follow naturally from an eigenvalue analysis of the (sparse) system matrix.

Boundary element methods (BEM) embody another important technique, and have been thoroughly discussed for multilayered media in the past [4], [5]. Their benefits are the natural simulation of open waveguides, thanks to the inherently satisfied radiation condition, and the need for fewer unknowns than a finite difference or finite element technique. The main drawback is the less trivial eigenmode analysis based on the search of complex zeros of the system matrix's determinant.

This contribution states important properties of the numerically obtained dispersion curves using a BEM. These properties are leveraged in a numerical implementation to yield an efficient eigenmode analysis of isotropic waveguides. A numerical example illustrates the eigenmode analysis of the fundamental modes of a pair of coupled microstrip lines on a finite substrate, and is compared with published results [6].

\section{GEOMETRY AND InTEGRAL EQUATIONS}

The waveguide cross section under study consists of connected homogeneous isotropic media $\Omega_{i}$, with permittivity $\epsilon_{i} \in \mathbb{C}$ and permeability $\mu_{i} \in \mathbb{C}$ (Fig. 1). There is one unbounded medium, called the background medium and denoted $\Omega_{0}$. The geometry is translation-invariant in the z-direction.

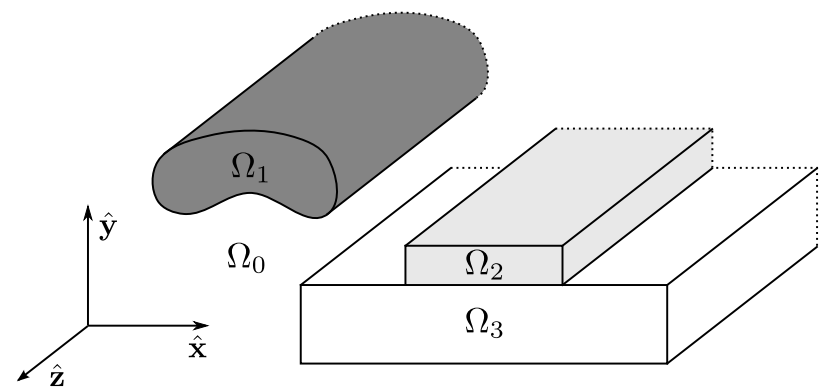

Fig. 1. Uniform waveguide with connected isotropic homogeneous domains $\Omega_{i}$. There is one unbounded background medium, denoted $\Omega_{0}$.

In an eigenmode analysis, we are interested in non-trivial solutions of the homogeneous time-harmonic Maxwell equations ( $e^{j \omega t}$ convention) that have an $e^{-j \beta z}$ dependence. This leads to representation formulas for the fields that depend on the parameters $\omega, \beta \in \mathbb{C}[1]$. Imposing continuity of the tangential fields at the medium boundaries, and introducing the two-dimensional version $\mathcal{M}$ of the PMCHWT operator [7]-[9], leads to the following system for the tangential traces of the fields:

$$
\mathcal{M}\left(\begin{array}{l}
\hat{\mathbf{n}} \times \mathbf{E} \\
\hat{\mathbf{n}} \times \mathbf{H}
\end{array}\right)=\mathbf{0} .
$$

The Green's function in medium $\Omega_{i}$ is given by $\frac{j}{4} H_{0}^{(2)}\left(\gamma_{i} r\right)$, with $r$ the distance between the source and observation point, $\gamma_{i}=\sqrt{\omega^{2} \epsilon_{i} \mu_{i}-\beta^{2}}$, and $H_{0}^{(2)}$ the zeroth order Hankel function of the second kind. For fixed $\omega$, the branch cuts of $\gamma_{i}$, denoted $\mathcal{B}_{i}(\omega)$, are chosen on the hyperbola in the $\beta$-plane such that $\Im \gamma_{i} \leq 0$, ensuring the radiation condition at infinity in the background medium.

\section{Properties OF THE DisCRETIZED SYSTEM}

The continuous operator $\mathcal{M}$ is discretized by restricting the unknown equivalent currents to a finite-dimensional sub- 
space, followed by testing the continuity equations using the method of moments (MoM). The MoM system matrix is denoted $\mathrm{M}(\omega, \beta)$, with $\mathfrak{D}(\omega, \beta)=\operatorname{det} \mathrm{M}(\omega, \beta)$. We call $\beta_{0}$ a propagation constant of the waveguide at the frequency $\omega_{0}$, if $\mathfrak{D}\left(\omega_{0}, \beta_{0}\right)=0$. A function $\beta(\omega)$, for $\omega \in(a, b)$, with $a, b \in \mathbb{R}$ and $\mathfrak{D}(\omega, \beta(\omega))=0$, is called a dispersion curve. The following theorems discuss the analyticity of the determinant and the behavior of a dispersion curve as a function of frequency [1].

Theorem III.1. $\mathfrak{D}(\omega, \beta)$ is holomorphic as a function of $\omega$ and $\beta$ in the domain $\mathcal{O}=\left(\mathbb{C}_{0} \times \mathbb{C}\right) \backslash\left(\cup_{i}\left\{(\omega, \beta) \mid \beta \in \mathcal{B}_{i}(\omega)\right\}\right)$.

Theorem III.2. Suppose that $\beta(\omega)$ is a complex-valued function of real argument $\omega \in\left(\omega_{0}-\epsilon, \omega_{0}\right]$, representing a zero of the determinant with constant multiplicity $\nu$, i.e. $\mathfrak{D}(\omega, \beta(\omega))=0$. Then there exists $a \delta>0$ such that $\beta(\omega)$ can be smoothly extended to the interval $\omega \in\left(\omega_{0}-\epsilon, \omega_{0}+\delta\right)$, still obeying $\mathfrak{D}(\omega, \beta(\omega))=0$ with multiplicity $\nu$. Therefore, dispersion curves cannot split up by themselves. There has to be at least an intersection with another curve or an intersection with a branch cut $\mathcal{B}_{i}(\omega)$.

Fig. 2 visualizes some implications of Theorem III.2. The bifurcation of the dispersion curve $\beta_{1}(\omega)$ at the frequency $\omega_{0}$ is possible, as this happens on the branch cut $\mathcal{B}_{i}\left(\omega_{0}\right)$. A physical example of this situation is the bifurcation of a mode into two complex modes [10]. However, the bifurcation of $\beta_{2}(\omega)$ at $\omega_{0}$ is not possible, as it would violate Theorem III.2.

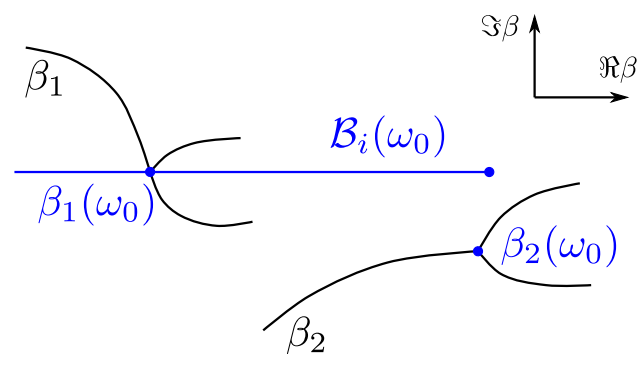

Fig. 2. Theorem III.2 implies that the bifurcation of the dispersion curve $\beta_{1}(\omega)$ at the frequency $\omega_{0}$ is possible, as it happens on the branch cut $\mathcal{B}_{i}\left(\omega_{0}\right)$. However, the bifurcation of $\beta_{2}(\omega)$ at $\omega_{0}$ is not possible.

The following theorem provides a connection between the multiplicity of a propagation constant and the dimension of the null space of the system matrix.

Theorem III.3. Suppose $\mathfrak{D}\left(\omega_{0}, \beta_{0}\right)=0$, i.e. $\beta_{0}$ is a zero at the frequency $\omega_{0}$ with multiplicity $\nu\left(\beta_{0}\right)$. Denote the nullity of the MoM matrix as $\nu\left(\mathrm{M}\left(\omega_{0}, \beta_{0}\right)\right)$. Then $\nu\left(\mathrm{M}\left(\omega_{0}, \beta_{0}\right)\right) \leq \nu\left(\beta_{0}\right)$.

Proof: $\operatorname{As~} \mathrm{M}\left(\omega_{0}, \beta\right)$ is an square matrix of dimension $n$ with holomorphic elements in a neighborhood of $\beta_{0}$ (see Theorem III.3 in [1]), it can there be expanded into the following Taylor series:

$$
\mathrm{M}\left(\omega_{0}, \beta\right)=\sum_{i=0}^{\infty} \mathrm{M}_{i}\left(\beta-\beta_{0}\right)^{i}
$$

with $\mathrm{M}_{i} \in \mathbb{C}^{n \times n}$ and, as $\beta_{0}$ is a zero of the determinant function, $\operatorname{det} \mathrm{M}_{0}=0$. Suppose the dimension of the null space of the matrix $\mathrm{M}_{0}$ is equal to $\nu_{0}$, i.e. $\nu_{0}=\nu\left(\mathrm{M}_{0}\right)=\nu\left(\mathrm{M}\left(\omega_{0}, \beta_{0}\right)\right)$. Now let us write the determinant function in the following form:

$$
\mathfrak{D}(\beta)=\operatorname{det}\left(\sum_{i=0}^{\infty} \mathrm{M}_{i}\left(\beta-\beta_{0}\right)^{i}\right)=\sum_{j=0}^{\infty} \alpha_{j}\left(\beta-\beta_{0}\right)^{j},
$$

with $\alpha_{j} \in \mathbb{C}$, with $\alpha_{0}=0$. Let us look at a term $\alpha_{j}(\beta-$ $\left.\beta_{0}\right)^{j}$ for $0<j<\nu_{0}$. It can be seen that $\alpha_{j}$ can be written as a sum of determinants of matrices $\mathrm{M}_{0}$ with $k \leq j$ rows replaced by corresponding rows of the matrices $\mathrm{M}_{i}$ (for $i>0$ ). Because only $k \leq j<\nu_{0}$ rows are replaced in this process, the untouched $n-k$ rows of $\mathrm{M}_{0}$ will be linearly dependent, implying $\alpha_{j}=0$ for $j<\nu_{0}$, and thus $\nu\left(\mathrm{M}\left(\omega_{0}, \beta_{0}\right)\right) \leq \nu\left(\beta_{0}\right)$.

\section{EFFICIENT NUMERICAL FrAMEWORK}

In this section, we present an efficient numerical framework for the eigenmode analysis of isotropic waveguides. The implementation is simplified after leveraging the stated theorems.

\section{A. Determination of the Propagation Constants}

In a first step, we determine the propagation constants of the waveguide at a fixed frequency $\omega_{0}>0$, in a rectangle $R=\left[\beta_{1}, \beta_{2}\right]$ that does not intersect a branch cut, i.e. $R \cap\left(\cup_{i} \mathcal{B}_{i}\left(\omega_{0}\right)\right)=\varnothing$. As $\mathfrak{D}\left(\omega_{0}, \beta\right)$ is holomorphic in $R$ (Theorem III.1), the number of propagation constants inside $R$ (counted with their multiplicity) is given by the following contour integral:

$$
N_{R}=\frac{1}{2 \pi j} \oint_{\partial R} \frac{\mathfrak{D}^{\prime}\left(\omega_{0}, \beta\right)}{\mathfrak{D}\left(\omega_{0}, \beta\right)} d \beta
$$

This contour integral can be reliably determined based on relatively few values of the determinant, using the argument principle [11]. If $N_{R}>0, R$ is subsequently partitioned into four rectangles for which (4) is again calculated, reusing the values of the determinant on $\partial R$. This procedure is iteratively applied until the rectangle is considered small enough. At that stage, we assume there is a single propagation constant in $R$, with multiplicity $\nu\left(\beta_{0}\right)=N_{R}$. Its location can be determined by calculating the following contour integral:

$$
\beta_{0}=\frac{1}{2 \pi \nu\left(\beta_{0}\right) j} \oint_{\partial R} \frac{\beta \mathfrak{D}^{\prime}\left(\omega_{0}, \beta\right)}{\mathfrak{D}\left(\omega_{0}, \beta\right)} d \beta
$$

This integral is numerically approximated with an adaptive Gauss-Kronrod quadrature rule.

\section{B. Determination of the Dispersion Curves}

The propagation constant $\beta_{0}$ with multiplicity $\nu\left(\beta_{0}\right)$ at the frequency $\omega_{0}$ is used as a seed value to calculate the dispersion curve $\beta(\omega)$ with $\beta\left(\omega_{0}\right)=\beta_{0}$, at the frequency points $\omega_{j}$, with $\omega_{i}<\omega_{j}$ and $0 \leq i<j \leq N$. Assuming that $\beta(\omega)$ crosses no branch-cut or other dispersion curve for $\omega \in\left[\omega_{0}, \omega_{N}\right]$, the dispersion curve is smooth (Theorem III.2). This allows to use an extrapolation method to estimate the location of 
the propagation constant at $\omega_{j}$, if the previously calculated values $\beta\left(\omega_{i}\right)$ are known, thus reducing the initial box $R$ of the previous section and thereby reducing calculation time. In the numerical implementation, an additional check $\nu\left(\beta\left(\omega_{j}\right)\right)=$ $\nu\left(\beta_{0}\right)$ is performed (Theorem III.2).

\section{Calculation of the Cross-sectional Eigenmode Profiles}

The cross-sectional eigenmode profiles, i.e. the electric and magnetic field distribution of the eigenmodes corresponding to a propagation constant $\beta_{0}$ at the frequency $\omega_{0}$, are calculated from the tangential boundary fields using representation formulas. The eigenvectors in the nullspace of $\mathrm{M}\left(\omega_{0}, \beta_{0}\right)$ are the expansion coefficients of the tangential traces of the eigenmodes on the material boundaries. The number of linearly independent eigenvectors is upper bounded by $\nu\left(\beta_{0}\right)$ (Theorem III.3).

\section{NUMERICAL EXAMPLE}

\section{A. Coupled Microstrip Lines on a Finite Substrate}

The presented method allows an efficient eigenmode analysis of a pair of coupled microstrip lines on a finite substrate and ground conductor (inset of Fig. 3). The two microstrip lines and the ground conductor have electric conductivity $\sigma=5.8 \cdot 10^{7}(\Omega \mathrm{m})^{-1}$, the substrate has relative permittivity $\epsilon_{r}=9.8$ and the background medium is free space. The dimensions of the waveguide are given by $d=1 \mathrm{~mm}$, $w=d / 0.635, t=0.3 w$ and $h=w$.

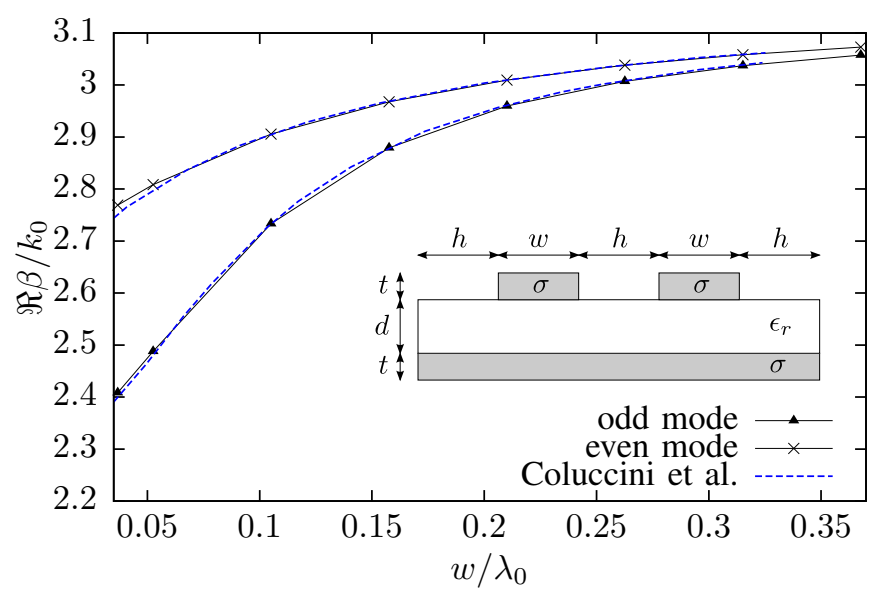

Fig. 3. Real part of the dispersion curves of the fundamental modes of the two coupled microstrip lines sketched in the inset. $d=1 \mathrm{~mm}, w=d / 0.635$ $t=0.3 w, h=w, \epsilon_{r}=9.8$ and $\sigma=5.8 \cdot 10^{7}(\Omega \mathrm{m})^{-1}$. Symbols: this method with copper conductors and finite substrate, dashed lines: Coluccini et al. [6] with perfect conductors and infinite substrate.

Fig. 3 shows the real part of the propagation constant of the two fundamental modes, normalized to the free space wave number $k_{0}=\omega \sqrt{\epsilon_{0} \mu_{0}}$, as a function of $w / \lambda_{0}=w f / c$, with $c$ the speed of light in vacuo and $f$ varying between $7 \mathrm{GHz}$ and $70 \mathrm{GHz}$. At high frequencies, the curves coincide with published results for the microstrip pair with perfect conductors and infinite substrate and ground plane [6]. The electric and magnetic field of the eigenmodes at high frequencies (Fig. 5

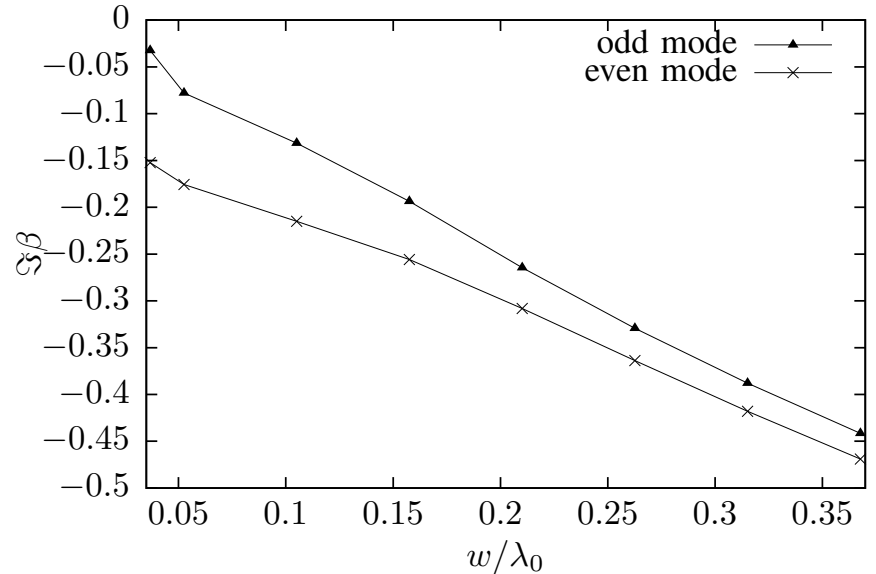

Fig. 4. Imaginary part of the dispersion curves of the fundamental modes of the two coupled microstrip lines.

and 6) are mostly confined in the substrate region directly below the microstrip lines, so that the finity of the substrate has no major influence on the propagation constants.

The imaginary part of the propagation constant of the two fundamental modes is shown in Fig. 4, and represents the waveguide losses that are caused by the finite conductivity of the conductors. For a waveguide of length $l$, the power loss of a propagating eigenmode is equal to $1-e^{2 l \Im \beta}$. Observe that the power loss increases with frequency, which is caused by two phenomena. First, the thickness of the skin effect layer in the conductors decreases with frequency, which increases the resistance per unit length ( $\sqrt{f}$ behavior). Second, at higher frequencies, the current in the microstrip lines is mostly flowing on the bottom edge and is less spread over the conductor boundary than at lower frequencies (this can be observed from the magnetic field distribution in Fig. 6), which further increases losses.

\section{CONCLUSiOnS}

This contribution presents analytic properties of the dispersion curves of isotropic piecewise homogeneous waveguides, obtained using a boundary element method. Taking these properties into account leads to an efficient numerical framework for the waveguide eigenmode analysis. A numerical example demonstrates the eigenmode analysis for a pair of coupled microstrip lines with finite electric conductivity on a finite substrate and is compared with published results.

\section{ACKNOWLEDGEMENTS}

The research of the first author is supported by a doctoral grant from the Research Foundation Flanders (FWO-V).

\section{REFERENCES}

[1] D. Dobbelaere, H. Rogier, and D. De Zutter, "Properties and numerical solutions of dispersion curves in general isotropic waveguides," Microwave Theory and Techniques, IEEE Transactions on, vol. 61, no. 9, pp. 3161-3168, 2013. 


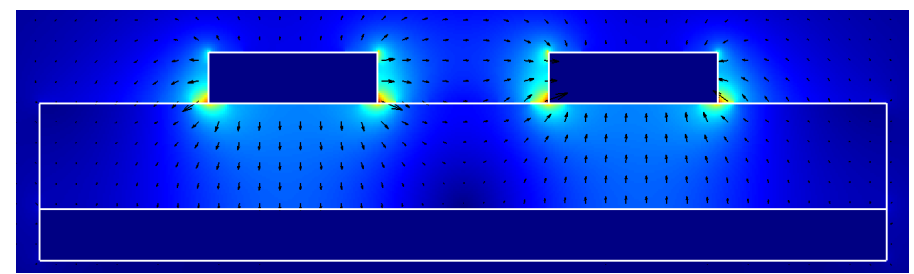

(a) Electric field of odd mode $(7 \mathrm{GHz})$

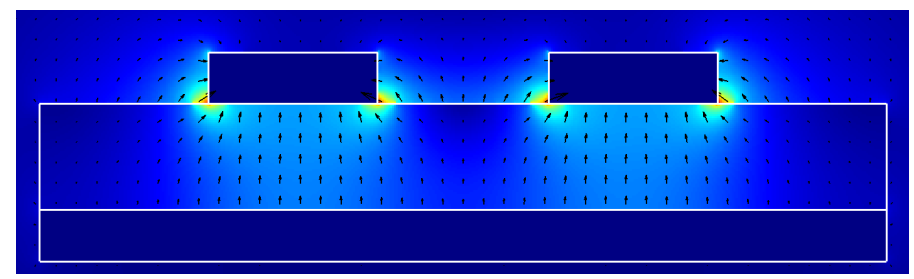

(b) Electric field of even mode $(7 \mathrm{GHz})$

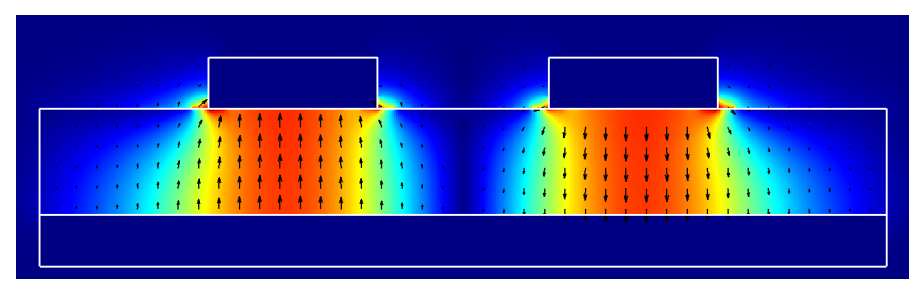

(c) Electric field of odd mode $(70 \mathrm{GHz})$

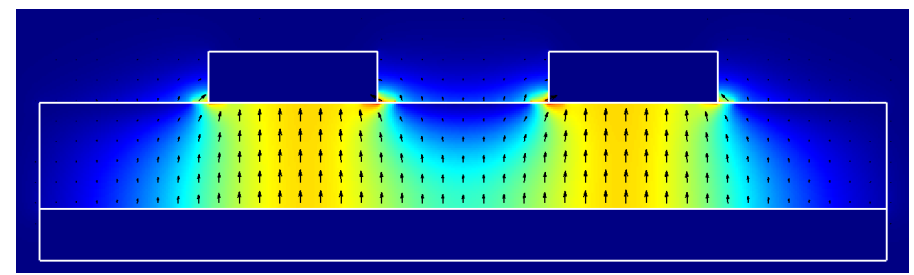

(d) Electric field of even mode $(70 \mathrm{GHz})$

Fig. 5. Cross-sectional tangential electric field distribution (normalized) of the two fundamental modes of the coupled microstrip lines at the frequencies $7 \mathrm{GHz}\left(w / \lambda_{0} \approx 0.037\right)$ and $70 \mathrm{GHz}\left(w / \lambda_{0} \approx 0.37\right)$.

[2] K. Bierwirth, N. Schulz, and F. Arndt, "Finite-difference analysis of rectangular dielectric waveguide structures," Microwave Theory and Techniques, IEEE Transactions on, vol. 34, no. 11, pp. 1104 - 1114, Nov. 1986.

[3] F. Fernandez, Y. Lu, J. Davies, and S. Zhu, "Finite element analysis of complex modes in inhomogeneous waveguides," Magnetics, IEEE Transactions on, vol. 29, no. 2, pp. 1601 -1604, Mar. 1993.

[4] F. Olyslager, D. De Zutter, and K. Blomme, "Rigorous analysis of the propagation characteristics of general lossless and lossy multiconductor transmission lines in multilayered media," Microwave Theory and Techniques, IEEE Transactions on, vol. 41, no. 1, pp. 79 -88, Jan. 1993.

[5] F. Olyslager and D. De Zutter, "Rigorous boundary integral equation solution for general isotropic and uniaxial anisotropic dielectric waveguides in multilayered media including losses, gain and leakage," Microwave Theory and Techniques, IEEE Transactions on, vol. 41, no. 8 , pp. 1385 -1392, Aug. 1993.

[6] G. Coluccini, M. Lucido, and G. Panariello, "Spectral domain analysis of open single and coupled microstrip lines with polygonal cross-section in bound and leaky regimes," Microwave Theory and Techniques, IEEE Transactions on, vol. 61, no. 2, pp. 736-745, 2013.

[7] A. Poggio and E. Miller, "Integral equation solution of three-dimensional scattering problems," in Computer Techniques for Electromagnetics, R. Mittra, Ed. Pergamon Press, 1973, ch. 4.

[8] Y. Chang and R. Harrington, "A surface formulation for characteristic modes of material bodies," Antennas and Propagation, IEEE Transactions on, vol. 25, no. 6, pp. $789-795$, Nov. 1977.

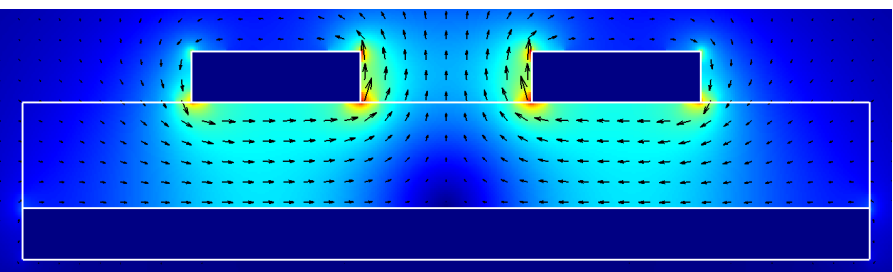

(a) Magnetic field of odd mode $(7 \mathrm{GHz})$

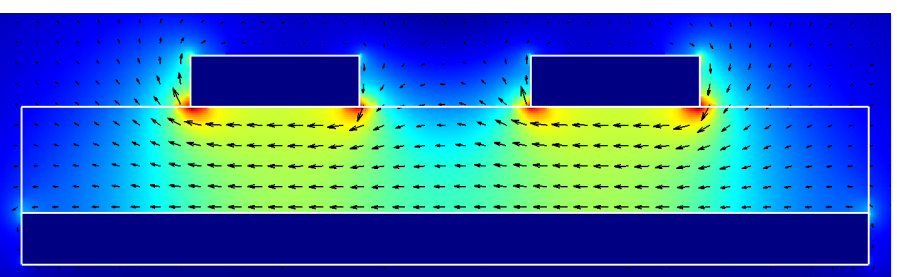

(b) Magnetic field of even mode $(7 \mathrm{GHz})$

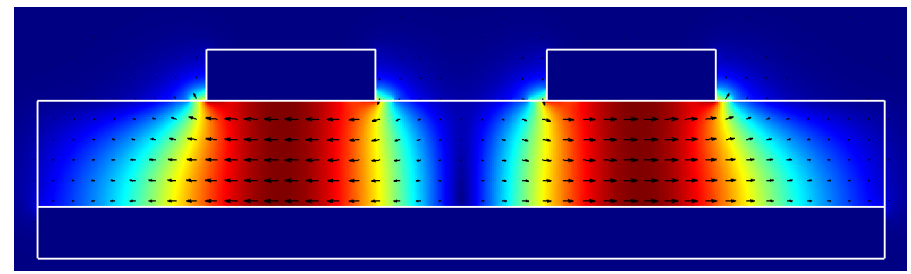

(c) Magnetic field of odd mode $(70 \mathrm{GHz})$

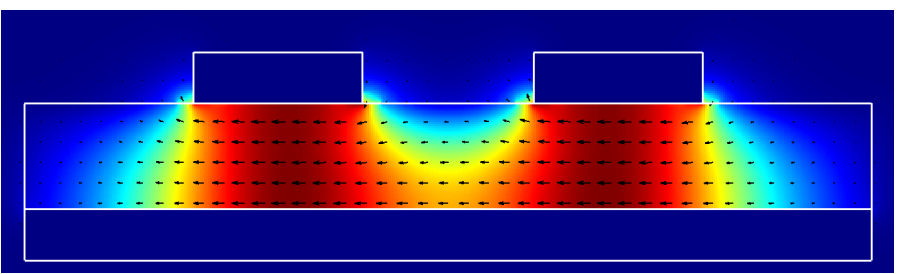

(d) Magnetic field of even mode (70 GHz)

Fig. 6. Cross-sectional tangential magnetic field distribution (normalized) of the two fundamental modes of the coupled microstrip lines at the frequencies $7 \mathrm{GHz}\left(w / \lambda_{0} \approx 0.037\right)$ and $70 \mathrm{GHz}\left(w / \lambda_{0} \approx 0.37\right)$.

[9] T. Wu and L. Tsai, "Scattering from arbitrarily-shaped lossy dielectric bodies of revolution," Radio Science, pp. 709-718, Sep. 1977.

[10] R. Islam and G. Eleftheriades, "On the independence of the excitation of complex modes in isotropic structures," Antennas and Propagation, IEEE Transactions on, vol. 58, no. 5, pp. 1567-1578, 2010.

[11] P. Henrici, Applied and Computational Complex Analysis, ser. Wiley Classics Library. Wiley, 1988. 